\title{
Robust Model for Multimodal Location of the Hazmat under Uncertainty
}

\author{
Liping Liu and Zipeng Yi* \\ School of Business, East China University of Science and Technology, Shanghai, 200237, China \\ *Corresponding author
}

\begin{abstract}
In reality, the demand of $O-D$ pair can be affected by various factors and then lead to uncertain demand. Finally, it will impactthe location of transfer yards. A bi-objective nonlinear robust model is established for locating multimodal transfer yards with hazardous materials in this paper. To solve easier by Cplex, a linear program is adopted. Finally, the developed model is applied to a numerical experiment, and the results are analyzed.
\end{abstract}

Keywords-hazmat; multimodal; uncertain demand; robust optimization; transfer yard location

\section{INTRODUCTION}

With the rapid development of the current industry, social chemical products constantly updated and the expansion of enterprise operation scope, the demand for hazmat increased year by year, so more and more companies began to engage in the transportation of hazmat. However, the transportation of hazmat will cause its movement. Meanwhile, the uncertain traffic and other road conditions willmake the occurrence of the risk further increases, so the transportation of hazmat should be paid more attention. In 1982, there are about 1.5billion tons of transportation of hazardous substance in US, accumulated mileage reached 784 billion miles (List and Abkowitz, 1986) [1]. In Virginia, hazmat transportation account for about $13 \%$ of the total transportation task (Schmidt and Price, 1979) [2].

As the trade volume of the world and transportation distance increased, single mode of transportation cannot meet the needs of all parts of the country, so in the past 20 years, more and more companies tend to choose multi-mode to complete the transportation task (i.e., multimodal transportation).However, hazmat transportation has the characteristics of low accident rate and great consequence. Therefore, for the characteristics of hazmat transportation, which can make full use of the advantages of multimodal to reduce the risk and cost of transportation. Multimodal transportation of hazmat requires the transfer of hazmat containersor tanks between different modes in transfer yard, the transfer process will further increase the transportation costs and risks. Due to the region and the surrounding population differences, the different location of transfer yard will cause different transfer risk and cost.

\section{LITERATURE REVIEW}

The research on multimodal transportation still focus on terminal or hub location and routing problem. Sörensen et al. (2012) studied the terminal location problem and established a model to minimize the total cost. The authors proposed two different metaheuristic procedures GRASP and ABHC to solve the problem, and the effectiveness of the two algorithms is compared [3].On the research of hub location problem, Alumur et al. (2012) studied the hub location problem from the perspective of network design with different possible transportation modes, and jointly consider transportation costs and travel times [4]. Ishfaq and Sox (2012) investigated the effect of limited hub resources on the design of intermodal logistics networks under service time requirements by integrating the hub operation queuing model and the hub location-allocation model [5]. Elhedhli and Wu (2010) also considered the congestion and queues in hub due to its capacity [6], the difference is the former considered the time delays due to the congestion and queues in hub, and then affected the service time and decision. The latter considered the congestion cost, and as part of the total cost, eventually influence the decision.

However, the problem of multimodal transportation for hazmat is different from the regular's. Transportation risk should be also considered. Most existing literatures about hazmat focus on the problem of routing optimization. Verma et al. (2012) studied the problem for rail-truckintermodal transportation of hazmat. A bi-objective model with minimize risk and cost is proposed, and an algorithm is developed to solve the problem [7].Assadipour et al. (2015) further considered congestion at intermodal yards and equipment capacity, and it will cause more risk and costs. They established a non-linear MIP model and solved by a multi-objectivegenetic algorithm [8].All above the literatures were deeply researched, but under deterministic scenario.

Most existing research of multimodal transportation is about multimodal location for regular goods or hazmat under deterministic case. In reality, the hazmat demand of OD pair is uncertain and its distribution is difficult to estimate. Therefore, themultimodal location of the hazmat under uncertainty is researched in this paper.

\section{Modeling}

In reality, the exact relation between each uncertainly source and the uncertain demand in terms of the probability distribution is often difficult to estimate. However, the robust optimization strategy is to immunize the transfer yard location decisions against the worst case demand uncertaintyrealization in the ellipsoidal uncertainty set (Shahabi and Unnikrishnan 2014), so we don't need to know its specific distribution [9]. 
Therefore, we apply the robust optimization solve the problem in this paper.

\section{A. Parameter and Variable Definition}

$M$ Set of OD pair, indexed by $m$.

$N$ Set of nodes in network, indexed by $\mathrm{i}$ and $\mathrm{j}$.

$K \quad$ Set of transportation modes, indexed by $\mathrm{k}$ and 1 .

$n^{m} \quad$ The number of shipments for the mth OD pair.

$r_{i j k}$ Risk due to moving one hazmat container from $i$ to $j$ use kth mode.

$r_{i k t}$ Risk due to transfer one hazmat container from mode $\mathrm{k}$ to mode 1 at node $\mathrm{i}$.

$C_{i j k}$ Cost due to moving one hazmat container from $\mathrm{i}$ to $\mathrm{j}$ use kth mode.

$C_{i k l}$ Cost due to transfer one hazmat container from mode $\mathrm{k}$ to mode 1 at node $\mathrm{i}$.

$f_{i} \quad$ Fixed costs of establishment and operating a transfer yard.

$$
\begin{gathered}
X_{i j k}^{m}=\left\{\begin{array}{l}
1, \text { if themth } O-D \text { pair transport fromi to } j \text { usemode } k \\
0, \text { otherwise }
\end{array}\right. \\
Y_{i}=\left\{\begin{array}{l}
1, \text { if thetransfer yard is established } \text { at node } i \\
0, \text { otherwise }
\end{array}\right. \\
Z_{i k l}^{m}=\left\{\begin{array}{l}
1, \text { if themth } O-D \text { pair transfer from mode } k \text { tol at node } i \\
0, \text { otherwise }
\end{array}\right.
\end{gathered}
$$

\section{B. A Bi-Objective Model under Deterministic Demand}

We first established a deterministic model. Because of the toxic, explosive characteristics of hazmat and the serious consequences of the hazmat transportation accidents to the public, it is necessary to consider the minimization of the total cost and risk of transportation. So the objective of the model is to minimize the total cost and risk, and establish a bi-objective model for multimodal location of the hazmat.

$$
\begin{aligned}
& \text { Obj1:R }=\min \sum_{m \in M} \sum_{i \in N} \sum_{j \in N} \sum_{k \in K} r_{i j k} n^{m} X_{i j k}^{m}+\sum_{m \in M} \sum_{i \in N} \sum_{k \in K} \sum_{l \in K, l \neq k} r_{i k l} n^{m} Z_{i k l}^{m} \\
& \text { Obj2:C }=\min \sum_{m \in M} \sum_{i \in N} \sum_{j \in N} \sum_{k \in K} C_{i j k} n^{m} X_{i j k}^{m}+\sum_{m \in M} \sum_{i \in N} \sum_{k \in K} \sum_{l \in K, I \neq k} C_{i k l} n^{m} Z_{i k l}^{m}+\sum_{i \in N} f_{i} Y_{i}
\end{aligned}
$$

s.t.

$$
\left\{\begin{array}{lll}
\sum_{j \in N} \sum_{k \in K} X_{i j k}^{m}-\sum_{j \in N} \sum_{k \in K} X_{j i k}^{m}=1 & \text { if } i=\operatorname{org}(m) & \forall m \in M, i \in N \\
\sum_{j \in N} \sum_{k \in K} X_{i j k}^{m}-\sum_{j \in N} \sum_{k \in K} X_{j i k}^{m}=-1 & \text { if } i=\operatorname{dest}(m) & \forall m \in M, i \in N \\
\sum_{j \in N} \sum_{k \in K} X_{i j k}^{m}-\sum_{j \in N} \sum_{k \in K} X_{j i k}^{m}=0 & \text { other } & \forall m \in M, i \in N \\
\sum_{j \in N} \sum_{k \in K} X_{i j k}^{m} \leq 1 & \forall m \in M, i \in N
\end{array}\right.
$$

$$
\begin{array}{cr}
\sum_{k \in K} \sum_{l \in K, l \neq k} Z_{i k l}^{m} \leq 1 & \forall m \in M, i \in N \\
\sum_{i \in N} X_{i j k}^{m}+\sum_{i \in N} X_{j i l}^{m} \leq 1+Z_{j k l}^{m} & \forall m \in M, j \in N, k, l \in K \\
\sum_{i \in N} X_{i j k}^{m}+\sum_{i \in N} X_{j i l}^{m}>Z_{j k l}^{m} & \forall m \in M, j \in N, k, l \in K \\
Z_{i k l(l \neq k)}^{m} \leq Y_{i} & \forall m \in M, i \in N, k, l \in K \\
X_{i j k}^{m} \in\{0,1\} & \forall m \in M, i, j \in N, k \in K \\
Y_{i} \in\{0,1\} & \forall i \in N \\
Z_{i k l}^{m} \in\{0,1\} & \forall m \in M, i \in N, k, l \in K
\end{array}
$$

The $O b j 1$ and $O b j 2$ represent the minimization of total risk and cost respectively. The Obj1 contains the risk of transportation and transshipment. The Obj2 include the transportation cost, transshipment cost, and the fixed cost to establish and operate transfer yards.

Constraint (1) is to ensure flow conservation; Constraint (2) states that any OD pair only can choose one transportation mode and one road at most; Constraint (3) represents that any OD pair only can transfer once at most in a node; Constraint (4) and constraint (5) ensure that the carrier transferred at the node $\mathrm{j}$ only they choose different transportation modes when arrive node $\mathrm{j}$ and then depart from it;Constraint (6) ensure that carrier transfer at a node $i$ is possible only if that transfer yard is installed. Constraint (7), (8) and (9) enforce the binary constraints on the decision variables.

\section{Robust Model for Multimodal Location of the Hazmat under Uncertainty}

The real demand can be described by mean value plus the perturbation value of disturbance factor. As follows:

$$
\tilde{n}^{m}=\bar{n}^{m}+\sum_{g \in G} b_{g}^{m} \tilde{\mu}_{g} \quad \forall m \in M
$$

where $\bar{n}^{m}$ is the mean demand of mth OD pair; $\tilde{\mu}_{g}$ represents the gth source of uncertainty which is an independent random variable, and $\left|\tilde{\mu}_{g}\right| \leq 1 ; b_{g}^{m}$ is the levels of demand uncertainty, which is weights associated with the gthvariable $\tilde{\mu}_{g}$.

The robust approachmakes the optimal decision at worst case to make the decision has robustness. To avoid the scenario of over conservative, the uncertain random variables $\tilde{\mu}_{g}$ are assumed to be limited to an ellipsoidal uncertainty set, i.e. $U=\left\{\tilde{\mu}_{g},\|\mu\| \leq \Omega\right\}$ (Shahabi and Unnikrishnan, 2014) where $\Omega$ is uncertainty budget [9]. To ensure the value of $\Omega$ is valid, the constraint of $\Omega \leq \sqrt{g}$ should be satisfied. We can build the bi-objective robust model as follows: 


$$
\begin{aligned}
& \text { Obj1 }=\text { Risk }: \min \left\{\max _{|\mu| \leqslant \Omega} \sum_{m \in M} \sum_{(i, j) \in \in N} \sum_{k \in K} r_{i j k}\left(\bar{n}^{m}+\sum_{g} b_{g}^{m} \tilde{\mu}_{g}\right) X_{i j k}^{m}+\right. \\
& \left.\max _{|\mu| \leqslant \Omega} \sum_{m \in M} \sum_{i \in N} \sum_{k \in K} \sum_{l \in K} r_{i k l}\left(\bar{n}^{m}+\sum_{g \in G} b_{g}^{m} \tilde{\mu}_{g}\right) Z_{i k l}^{m}\right\} \\
& \text { Obj2 }=\operatorname{Cost}: \min \left\{\max _{|\mu| \leqslant \Omega} \sum_{m \in M} \sum_{(i, j) \in N} \sum_{k \in K} C_{i j k}\left(\bar{n}^{m}+\sum_{g} b_{g}^{m} \tilde{\mu}_{g}\right) X_{i j k}^{m}+\right. \\
& \left.\max _{|\mu| \leq \Omega} \sum_{m \in M} \sum_{i \in N} \sum_{k \in K} \sum_{l \in K} C_{i k l}\left(\bar{n}^{m}+\sum_{g} b_{g}^{m} \tilde{\mu}_{g}\right) Z_{i k l}^{m}+\sum_{i \in N} f_{i} Y_{i}\right\}
\end{aligned}
$$

s.t. $(1) \sim(9)$.

\section{SOLUTION PROCEDURE}

Since the maximization problem is based on the random variable $\tilde{\mu}_{g}$, the objective function $O b j 1$ and $O b j 2$ can be rewritten as follows:

$$
\begin{aligned}
& \text { Obj1 }=\text { Risk }: \min \left\{\left[\sum_{m \in M} \sum_{(i, j) \in N} \sum_{k \in K}\left(\bar{n}^{m} r_{i j k} X_{i j k}^{m}+\max _{|\mu| \mid \leq \Omega} \sum_{g \in G} b_{g}^{m} \tilde{\mu}_{g} r_{i j k} X_{i j k}^{m}\right)\right]+\right. \\
& \left.\left[\sum_{m \in M} \sum_{i \in N} \sum_{k \in K} \sum_{l \in K, l \neq k}\left(\bar{n}^{m} r_{i k l} Z_{i k l}^{m}+\max _{\| k \mid \leqslant \Omega} \sum_{g \in G} b_{g}^{m} \tilde{\mu}_{g} r_{i k l} Z_{i k l}^{m}\right)\right]\right\} \\
& \text { Obj2 = Cost }: \min \left\{\left[\sum_{m \in M} \sum_{(i, j) \in N} \sum_{k \in K}\left(\bar{n}^{m} C_{i j k} X_{i j k}^{m}+\max _{\|\mu\| \leq \Omega} \sum_{g \in G} b_{g}^{m} \tilde{\mu}_{g} C_{i j k} X_{i j k}^{m}\right)\right]+\right. \\
& \left.\left[\sum_{m \in M} \sum_{i \in N} \sum_{k \in K} \sum_{l \in K, \mid \neq k}\left(\bar{n}^{m} C_{i k l} Z_{i k l}^{m}+\max _{\| \mu \mid \leqslant \Omega} \sum_{g \in G} b_{g}^{m} \tilde{\mu}_{g} C_{i k l} Z_{i k l}^{m}\right)\right]+\sum_{i \in N} f_{i} Y_{i}\right\}
\end{aligned}
$$

The min-max formulation is a mixed integer nonlinear program which is difficult to solve, so it can be divided into two steps:

First, the inner maximization problem can be written as $\mathrm{S} 1$ and S2 in Eq. (15) and (16).

$$
\begin{aligned}
& S 1=\max _{\|\mu\| \leq \Omega} \sum_{m \in M} \sum_{i \in N} \sum_{k \in K} \sum_{g \in G} b_{g}^{m} \tilde{\mu}_{g}\left(\sum_{j \in N} r_{i j k} X_{i j k}^{m}+\sum_{l \in K} r_{i k l} Z_{i k l}^{m}\right) \\
& S 2=\max _{\|\mu\| \leq \Omega} \sum_{m \in M} \sum_{i \in N} \sum_{k \in K} \sum_{g \in G} b_{g}^{m} \tilde{\mu}_{g}\left(\sum_{j \in N} C_{i j k} X_{i j k}^{m}+\sum_{l \in K} C_{i k l} Z_{i k l}^{m}\right)
\end{aligned}
$$

To solve the maximization problem of $\mathrm{S} 1$, a lagrangian relaxation scheme is used to determine the optimal solutionexpression for the primitive uncertainty variables $\tilde{\mu}_{g}$ (Gülpınar et al., 2013) [10], the same to S2.

Considering the Lagrangian multiplier $\lambda \geq 0$, and $\|\mu\| \leq \Omega$, the Lagrangian function can be written as Eq. (17).

$$
L(\tilde{\mu}, \quad \lambda)=\sum_{m \in M} \sum_{i \in N} \sum_{k \in K} \sum_{g \in G} b_{g}^{m} \tilde{\mu}_{g}\left(\sum_{j \in N} r_{i j k} X_{i j k}^{m}+\sum_{l \in K} r_{i k l} Z_{i k l}^{m}\right)+\lambda(\Omega-\|\tilde{\mu}\|)
$$

The first order condition for the Lagrangian functionis given as:

$$
\left\{\begin{array}{l}
\frac{\partial L(\tilde{\mu}, \lambda)}{\partial \tilde{\mu}_{g}}=\sum_{m \in M} \sum_{i \in N} \sum_{k \in K} b_{g}^{m}\left(\sum_{j \in N} r_{j k} X_{i j k}^{m}+\sum_{l \in K} r_{i k l} Z_{i k l}^{m}\right)-\lambda \frac{\tilde{\mu}_{g}}{\|\tilde{\mu}\|}=0 \quad \forall g \in G \quad \text { (18) } \\
\frac{\partial L(\tilde{\mu}, \lambda)}{\partial \lambda}=\Omega-\|\tilde{\mu}\|
\end{array}\right.
$$

From above conditions, we can get the values of $\lambda$ and $\tilde{\mu}_{g}:$

$$
\left\{\begin{array}{l}
\lambda=\sqrt{\sum_{g \in G}\left[\sum_{m \in M} \sum_{i \in N} \sum_{k \in K} b_{g}^{m}\left(\sum_{j \in N} r_{i j k} X_{i j k}^{m}+\sum_{l \in K} r_{i k l} Z_{i k l}^{m}\right)\right]^{2}} \\
\tilde{\mu}_{g}=\Omega \frac{\sum_{m \in M} \sum_{i \in N} \sum_{k \in K} b_{g}^{m}\left(\sum_{j \in N} r_{i j k} X_{i j k}^{m}+\sum_{l \in K} r_{i k l} Z_{i k l}^{m}\right)}{\sqrt{\sum_{g \in G}\left[\sum_{m \in M} \sum_{i \in N} \sum_{k \in K} b_{g}^{m}\left(\sum_{j \in N} r_{i j k} X_{i j k}^{m}+\sum_{l \in K} r_{i k l} Z_{i k k}^{m}\right)\right]^{2}}} \forall g \in G
\end{array}\right.
$$

Second, plug the optimal value of $\tilde{\mu}_{g}$ into $\mathrm{S} 1$, the maximization problem of S1 can be solved in Eq. (22).

$$
S 1=\Omega \sqrt{\sum_{g \in G}\left[\sum_{m \in M} \sum_{i \in N} \sum_{k \in K} b_{g}^{m}\left(\sum_{j \in N} r_{i j k} X_{i j k}^{m}+\sum_{l \in K} r_{i k l} Z_{i k k}^{m}\right)\right]^{2}}
$$

The same to $\mathrm{S} 2$ in Eq. (23).

$$
S 2=\Omega \sqrt{\sum_{g \in G}\left[\sum_{m \in M} \sum_{i \in N} \sum_{k \in K} b_{g}^{m}\left(\sum_{j \in N} C_{i j k} X_{i j k}^{m}+\sum_{l \in K} C_{i k l} Z_{i k l}^{m}\right)\right]^{2}}
$$

Incorporating the solution of the inner maximization problems of S1 and S2 into Eq. (15) and (16), the min-max formulation for the robust multimodal location problem can be converted to the following minimization problem.

$$
\begin{aligned}
& \text { Obj1 }=\text { Risk: } \min \sum_{m \in M} \sum_{i \in N} \sum_{j \in N} \sum_{k \in K} \bar{n}^{m} r_{i j k} X_{i j k}^{m}+\sum_{m \in M} \sum_{i \in N} \sum_{k \in K} \sum_{l \in K} \bar{n}^{m} r_{i k l} Z_{i k l}^{m}+ \\
& \Omega \sqrt{\sum_{g \in G}\left[\sum_{m \in M} \sum_{i \in N} \sum_{k \in K} b_{g}^{m}\left(\sum_{j \in N} r_{i j k} X_{i j k}^{m}+\sum_{l \in K} r_{i k l} Z_{i k l}^{m}\right)\right]^{2}} \\
& \text { Obj2 }=\operatorname{Cost}: \min \sum_{m \in M} \sum_{i \in N} \sum_{j \in N} \sum_{k \in K} \bar{n}^{m} C_{i j k} X_{i j k}^{m}+\sum_{m \in M} \sum_{i \in N} \sum_{k \in K} \sum_{l \in K} \bar{n}^{m} C_{i k} Z_{i k l}^{m}+\sum_{i \in N} f_{i} Y_{i}+ \\
& \Omega \sqrt{\sum_{g \in G}\left[\sum_{m \in M} \sum_{i \in N} \sum_{k \in K} b_{g}^{m}\left(\sum_{j \in N} C_{i j k} X_{i j k}^{m}+\sum_{l \in K} C_{i k l} Z_{i k l}^{m}\right)\right]^{2}}
\end{aligned}
$$

Both the objective functions are nonlinear and complicated, to solve easier, $b_{g}^{m}$ can be written as $b_{g}^{m}=\bar{n}^{m} b_{g}$, and the $O b j 1$ and Obj2 can be reformulated as follows, which can be solved efficiently using CPLEX.

$$
\begin{aligned}
& \text { Objl=Risk: } \min \left[\sum_{m \in M} \sum_{i \in N} \sum_{j \in N} \sum_{k \in K} \bar{n}^{m} r_{i j k} X_{i j k}^{m}+\sum_{m \in M} \sum_{i \in N} \sum_{k \in K} \sum_{l \in K} \bar{n}^{m} r_{i j l} Z_{i k l}^{m}\right]\left[1+\Omega \sqrt{\sum_{g \in G} b_{g}^{2}}\right]
\end{aligned}
$$

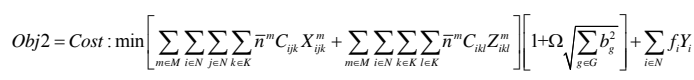

S.t.(1) (9).

Due to the bi-objective model is difficult to solve because of the incoordination of the target dimension, the two objective functions must be dimensionless unified treatment and transformed into a single objective function:

$$
O b j 3=\alpha \frac{O b j 1-O b j 1^{*}}{O b j 1^{*}}+(1-\alpha) \frac{O b j 2-O b j 2 *}{O b j 2 *}
$$

where $\alpha$ is the weight of risk, Obj1* and $O b j 2 *$ are the respective optimal value of objective functions $O b j 1$ and Obj2. 
So the bi-objective model can be transformed into following formulation:

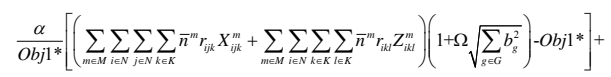

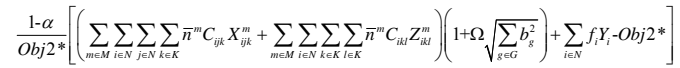

s.t. (1) (9).

The above model is a single objective mixed integer conic quadratic program which can be solved efficiently by CPLEX.

\section{REFERENCES}

[1] List, G., Abkowitz, M., 1986. Estimates of current hazardous materials flow patterns[J]. Transportation Quarterly, 40(4).

[2] Schmidt, J. W., Price, D. L., 1979. The flow of hazzardous materials on Virginia highways[J]. Journal of safety research, 11(3): 109-120.

[3] Sörensen, K., Vanovermeire, C., Busschaert, S., 2012. Efficient metaheuristics to solve the intermodal terminal location problem[J]. Computers \& Operations Research, 39(9): 2079-2090.

[4] Alumur, S. A., Kara, B. Y., Karasan, O. E., 2012. Multimodal hub location and hub network design[J]. Omega, 40(6): 927-939.

[5] Ishfaq, R., Sox, C. R., 2012. Design of intermodal logistics networks with hub delays[J]. European Journal of Operational Research, 220(3): 629-641.

[6] Elhedhli, S., Wu, H., 2010. A Lagrangean heuristic for hub-and-spoke system design with capacity selection and congestion[J]. INFORMS Journal on Computing, 22(2): 282-296.

[7] Verma, M., Verter, V., Zufferey, N., 2012. A bi-objective model for planning and managing rail-truck intermodal transportation of hazardous materials $[\mathrm{J}]$. Transportation research part E: logistics and transportation review, 48(1): 132-149.

[8] Assadipour, G., Ke, G. Y., Verma, M., 2015. Planning and managing intermodal transportation of hazardous materials with capacity selection and congestion[J]. Transportation Research Part E: Logistics and Transportation Review, 76: 45-57.

[9] Shahabi, M., Unnikrishnan, A., 2014. Robust hub network design problem[J]. Transportation Research Part E: Logistics and Transportation Review, 70: 356-373.

[10] Gülpınar, N., Pachamanova, D., Çanakoğlu, E., 2013. Robust strategies for facility location under uncertainty[J]. European Journal of Operational Research, 225(1): 21-35. 